Indian J Anim Health (2021), 60(2)-Special Issue: 213-220

DOI: https://doi.org/10.36062/ijah.2021.spl.00321

\title{
Isolation and phenotypic identification of Campylobacter in dairy supply chain
}

\author{
S. Bade ${ }^{1}$, D. Bhardwaj ${ }^{1}$, A. Jaswal ${ }^{1}$, H. M. Rashmi ${ }^{1}$, N. Kumar ${ }^{1}$ and H. V. Raghu ${ }^{1 *}$ \\ ${ }^{1}$ Dairy Microbiology Division, ICAR-National Dairy Research Institute, Karnal - 132 001, Haryana, \\ India
}

\begin{abstract}
A total of 175 samples comprising of raw milk (30), freshwater (30), dairy wastewater (30), Human handler swab (30), Milk filter swab (30), and faecal swab (25) samples were collected from respective sources for the isolation of Campylobacter species. Among 175 samples, 53 samples were selected based on its positive growth in selective enrichment and characteristics colonies on mCCDA media. From 53 positive samples, 91 colonies showed characteristics purple to mauve colour colonies on Hi-Chrome media. Among 91 isolates selected, 41 isolates have shown pink-coloured curved rods/s-shaped rods/cocci's by Gram's staining (G -ve) and 3 isolates among these were identified as Campylobacter based on biochemical tests such as oxidase, catalase, and microaerobic growth at $25^{\circ} \mathrm{C}$. Later, all 3 isolates were evaluated for the $\mathrm{H}_{2} \mathrm{~S}$ production and Hippurate hydrolysis test wherein 2 isolates showed positive hippurate hydrolysis test and negative $\mathrm{H}_{2} \mathrm{~S}$ production and in another isolate has shown negative Hippurate hydrolysis test and positive $\mathrm{H}_{2} \mathrm{~S}$ production. Based on the Hippurate hydrolysis test and $\mathrm{H}_{2} \mathrm{~S}$ production these isolates were tentatively considered as $C$. jejuni (2 nos) and C. coli (1 no). These isolates were mainly originated from raw milk and faecal swab samples and none of the other samples have shown any presence of Campylobacter spp. among the samples screened. The occurrence rate of Campylobacter spp. was found more in faecal swab samples as compared to raw milk samples and it was mainly due to its origin of its presence in faecal samples.
\end{abstract}

Key words: Campylobacter, Dairy, Identification, Isolation, Supply chain

\section{Highlights}

- In total, 175 samples from different dairy supply chains were used to isolate Campylobacter species.

- About 91 isolates grew on mCCDA and Hi-Chrome media, showing purple to mauve colored colonies.

- Based on a Hippurate hydrolysis test and $\mathrm{H}_{2} \mathrm{~S}$ production, 3 isolates were tentatively confirmed to be Campylobacter species

- These isolates originated mainly from raw milk and fecal swabs.

\section{INTRODUCTION}

Like food safety, consumers are now looking for quality too. The food-borne infection which caused due to microorganisms increasing day by day and the public health burden due to diseases caused by them also increasing since perishable foods such as milk and milk products are susceptible to microbial contamination under the unfavourable conditions (hot, humid) mainly during the processing and marketing operations in

developing countries like India. Raw milk may be consumed at the farm or it may be sold directly to the market. In Poland, the selling of raw milk is legal as in the other EU countries. Besides, the hygiene regulations applicable to food of animal origin permit the selling of only the milk containing no more than 100,000 microorganisms per $1 \mathrm{~mL}$ originating from farms under veterinary supervision and meeting the systemic sanitary criteria in Poland (MARD,

*Corresponding Author, E Mail: Raghu.V@icar.gov.in ,4rvsy.dmndri@gmail.com 
2007). As we see the above requirements, we can't assume or do not guarantee the safety for the consumption of raw milk, it may be hazardous when consumed without pasteurization or any processing.

Raw milk is a source of food for various microorganisms because it contains a lot of nutrients. The pathogens like Listeria monocytogenes, Salmonella spp., Campylobacter spp., Enterobacter spp., E. coli, etc. has been found in raw milk. Among this Campylobacter species outbreaks in raw milk have been increasing, mainly in European Countries (Bintsis, 2017). Diseases like gastritis and enteritis are caused due to the consumption of raw milk (Dhanashekar et al., 2012). The Campylobacter spp. are micro-aerophilic, spiral in shape, Gram-negative bacteria, the primary cause of bacterial diarrhea in humans in the United States, reported at 1.3 million cases, and 120 deaths per year (Kaakoush et al., 2015). In humans, Campylobacter gastroenteritis varies from moderate to serious diarrheal disease (Kaakoush et al., 2015). The infection caused due to Campylobacter jejuni is called campylobacteriosis, or Campylobacter jejuni gastroenteritis. Infection primarily occurs in old people, infants, and immunocompromised patients (Same and Tamma, 2018). The person who has been infected with the organism from contaminated food or water is at risk of contracting the disease (Bintsis, 2017). The individuals who are immunocompromised or sick are mostly at higher risk of contracting campylobacteriosis and the infection is also more severe in those individuals. Campylobacteriosis is usually considered as a self-limiting disease, and there is usually no evidence of antimicrobial therapy for treating this infection (Barr and Smith, 2014). However, if it is started early in the course of infection on very sick or immunocompromised patients, the treatment can delay or decrease the length and impact or severity of the illness. Complications are rare, but Campylobacter jejuni infection can cause serious sequelae such as reactive arthritis, meningitis, recurrent colitis, acute cholecystitis, haemolytic uremic syndrome, pancreatitis, cystitis, and Guillain-Barre syndrome. From a food safety point of view, Campylobacter jejuni and Campylobacter coli are most significant and cause enteritis in human beings as well as livestock (Facciolà et al., 2017).

Campylobacters are the intestinal inhabitants of a wide variety of wild and domestic animals, primarily birds. Insufficiently cooked meat, particularly poultry, unpasteurized milk, contaminated drinking water, direct contact with animals, fecal contamination of domestic animals, and surface water contaminating birds act as the primary source of the organism (Igwaran and Okoh, 2019). However, there is also over contamination of milk as a result of mastitis. It causes animals to suffer from diarrhoea and abortion. The occurrence of these Campylobacter species has been identified in bulk tank milk, dairy filters, manure, and animal faeces. In India there is very little information available on the occurrence of Campylobacter mainly in the dairy supply chain as a source of the pathogen, due to fewer detection practices (Sippy et al., 2012). As no systematic monitoring of Campylobacter in milk, as well as milk products is conducted, the public health burden due to campylobacteriosis is also growing today (Facciolà et al., 2017). So there is a need for the routine analysis of this pathogen in the dairy supply chain to know the chances of infection of Campylobacter by consumption of milk and milk products. Therefore this study was conducted to determine the occurrence of Campylobacter in the study area by isolating Campylobacter from dairy supply chain.

\section{MATERIALS AND METHODS}

Procurement and maintenance of culture: The reference cultures required for our study $C$. coli ATCC 33559 and $C$. jejuni ATCC 29428 were procured from American type culture collection and checked for purity. Cultures were activated in Brucella broth (M348, Himedia, Mumbai, India) followed by streaking on modified 
charcoal cefoperazone deoxycholate agar (mCCDA, M8871, Himedia, Mumbai, India), and Campylobacter agar base (CAB, M994, Mumbai, India), followed by incubation at $37^{\circ} \mathrm{C}$ for 24-48 h. A single pure colony from respective selective media after microscopic examination was picked up and maintained on Brucella broth or Bolton broth by routine subculturing after every $15^{\text {th }}$ day. All the experiments were conducted using overnight grown cultures.

Sampling: For the collection of samples from the cattle-yard, initially, the hands of the milking man were sanitized using $70 \%$ alcohol. Several streams of milk were discarded from all four teats as foremilk. After that samples were collected in $100 \mathrm{~mL}$ sterile sample bottle directly from all quarters of the teats. There was a total of 175 samples among which 30 raw milk, 30 fresh water, 30 dairy waste water, 30 human handler swab samples, 30 milk filter swab, and 25 faecal swab samples were collected from Live stock research centre (LRC) and Experimental dairy plant, ICAR-NDRI, Karnal, Haryana. After aseptic collection, samples were labelled and packed with sterile bottles and transported with an ice box to the microbiology section of National Referral Centre for Milk Quality and Safety, ICARNDRI, Karnal for bacterial isolation. Samples were processed immediately for bacterial identification to species level using culture media and then isolates were kept in refrigerator at $4^{\circ} \mathrm{C}$ until microbial characterization with regular sub-culturing. Sampling of human handler and faecal swab was collected by swab techniques followed by evaluation using conventional technique.

Isolation of Campylobacter species from milk and water samples: Initially, the ATCC culture of Campylobacter jejuni and Campylobacter coli were evaluated. Samples were processed to isolate the Campylobacter spp. as per the ISO (10272-1-2006) protocol. Bolton broth was used for the selective enrichment of Campylobacter species. Before use, the Bolton broth was autoclaved at $121{ }^{\circ} \mathrm{C}$ at $15 \mathrm{lbs}$. pressure for 15 minutes. After cooling to $50^{\circ} \mathrm{C}$, the rehydrated contents of Bolton selective supplement was added followed by $5 \%$ defibrinated sheep blood, mixed well separately and aseptically dispensed into the tubes. The tubes were marked respective to their sample numbers and $1 \mathrm{~mL}$ of samples (milk and water) inoculated in $9 \mathrm{~mL}$ of Bolton broth. The inoculated tubes were incubated at $37^{\circ} \mathrm{C}$ for 4 $6 \mathrm{~h}$ and then at $41.5^{\circ} \mathrm{C}$ for $44 \mathrm{~h}$ in the microaerophilic condition which was created using 'CampyGen' gas pack in an anaerobic jar. The supplement added to the medium contains different antibiotics such as vancomycin, cefoperazone, and trimethoprim for better selectivity. Modified charcoal cefoperazone deoxycholate agar base (mCCDA) was recommended for selective detection and enumeration of Campylobacter species (Smith et al., 2015). Further, enrichment was done by plating on blood free modified charcoal cefoperazone deoxycholate agar (mCCDA). The inoculated plates were incubated at a microaerophilic environment $\left(85 \% \mathrm{~N}_{2}, 5 \% \mathrm{O}_{2}\right.$, and $\left.10 \% \mathrm{CO}_{2}\right)$ at $41.5^{\circ} \mathrm{C}$ for 24-48 h. The typical dewdrop like, greyish, flat colonies on this blood-free agar were isolated. The microaerophilic environment was created in an anaerobic jar by using the 'CampyGen' gas pack and the CCDA supplement containing antibiotics was also used with mCCDA to increase the selectivity of media. The characteristics colonies were further transferred into Brucella broth and incubated at $37^{\circ} \mathrm{C}$ for $24 \mathrm{~h}$. subsequently, these isolates were stored in $40 \%$ glycerol and kept at $-20{ }^{\circ} \mathrm{C}$ until further investigation.

Isolation of Campylobacter isolates from human handlers, dairy milk filters, and faecal swab samples: Ten $\mathrm{mL}$ of ringer's solution tubes were prepared and autoclaved at $121^{\circ} \mathrm{C}$ for 15 mins at 15 psi pressure. The sterile cotton swab was dipped into the ringers solution tubes so that it will get wet. After that swabs were taken out from the tubes and rubbed on the hands of humans working in the dairy chain and a similar 
procedure was followed for dairy milk filters and faecal swab samples, and transferred back into the tubes. One $\mathrm{mL}$ of sample from the ringer's tube was transferred into an autoclaved $9 \mathrm{~mL}$ of Bolton broth tube. All the tubes were marked for their identification and kept at $37^{\circ} \mathrm{C}$ for $4-6 \mathrm{~h}$ and $41.5^{\circ} \mathrm{C}$ for $44 \mathrm{~h}$ in microaerophilic conditions. Tubes which were showed turbidity were streaked on modified charcoal cefoperazone deoxycholate agar (mCCDA) plates followed by incubation at $41.5^{\circ} \mathrm{C}$ for $24-$ $48 \mathrm{~h}$ in microaerophilic condition. The characteristics of greyish, flat colonies are selected and picked for further biochemical confirmation. In the case of dairy milk filters and faecal samples, the samples were taken from different locations.

\section{Identification of positive Campylobacter} isolates by phenotypic methods: The characteristics of greyish, flat, and dewdrop like colonies were further evaluated for its confirmation using different identification tests such as Grams staining, oxidase test, catalase test, micro-aerobic growth at $25^{\circ} \mathrm{C} \& 42^{\circ} \mathrm{C}$ and $\mathrm{H}_{2} \mathrm{~S}$ test, etc. as per ISO 10272-1-2006 method.

Hippurate hydrolysis test: The sodium hippurate broth was prepared and autoclaved at $121^{\circ} \mathrm{C}$ at $15 \mathrm{lbs}$ pressure for 15 minutes and inoculated with overnight grown culture. The sample was then incubated at $41.5^{\circ} \mathrm{C}$ for $24 \mathrm{~h}$ under microaerophilic conditions. The broth was centrifuged and the precipitate was removed. The prepared ferric chloride reagent was added to the supernatant. If the precipitate remains after 10 minutes, benzoic acid is present and the test was considered positive for hippurate hydrolysis (Mitruka, 2017).

\section{RESULTS}

Selection of selective media for isolation of Campylobacter: During the selection of selective media for the isolation of Campylobacter, two different selective media such as blood containing agar CAB (Campylobacter agar base) and blood-free agar modified charcoal cefoperazone deoxycholate agar (mCCDA) were screened using standard ATCC cultures of Campylobacter coli (33559) and Campylobacter jejuni (29428) for better isolation of Campylobacter species. As the mCCDA contained activated charcoal and was blood-free, it was shown better selectivity and the chances of contamination were also less than blood containing media ' $\mathrm{CAB}$ '. The characteristics of colonies of C. coli and C. jejuni are shown in Fig. 1.

Isolation of Campylobacter from dairy supply chain: Out of 175 total samples, 30 samples of raw milk including 12 buffalo milk and 18 cow milk samples were processed for isolation of Campylobacter species. Out of 30 raw milk samples, 12 samples showed positive growth in selective enrichment, and characteristics of greyish colonies were also observed on mCCDA. The characteristics colonies from these 12 samples were further streaked on HiChrome Campylobacter agar base and 25 colonies had shown characteristic mauve to purple-colored colonies. In case of freshwater and dairy wastewater, thirty samples each from freshwater and dairy wastewater were processed for isolation of Campylobacter species. Thirty samples from freshwater and 9 from dairy wastewater were showing positive growth on selective enrichment and characteristics growth on mCCDA. Further, these samples were streaked on HiChrome $\mathrm{CAB}$ and 6 characteristic colonies from freshwater and 13 from dairy wastewater were selected for further confirmation. Human handler swab samples, 10 among 30 samples had shown positive growth in selective enrichment, and characteristics of greyish colonies on mCCDA medium. The characteristics colonies on these 10 samples were further streaked on HiChrome Campylobacter agar base, only 2 isolates had shown characteristic mauve to purple-colored colonies. Five samples among milk filter swabs had shown positive growth in selective enrichment, and characteristic greyish colonies on mCCDA medium. The characteristics colonies on these 05 samples were further streaked on HiChrome Campylobacter agar 
base and 10 isolates had shown characteristic mauve to purple-colored colonies. Fourteen samples among 25 samples of faecal swab samples had shown positive growth in selective enrichment, and characteristic greyish colonies on mCCDA medium. All the characteristics colonies isolated from 175 numbers of samples were further streaked on HiChrome Campylobacter agar base and 91 isolates showed characteristic mauve to purple-colored colonies from 53 samples (Fig. 2).

\section{Identification of positive Campylobacter} isolates by phenotypic methods: All 91 isolates were further screened by Grams staining. Out of 91 isolates, 41 were observed as Gramnegative curved rods/s-shaped/coccoid in shape (Fig. 3A). Out of 25 raw milk isolates, 13 were confirmed as gram-negative rods. Similarly, 2 isolates from freshwater, 5 from dairy wastewater, 17 from dairy milk filter, and 17 from faecal swab sample were confirmed by Gram's staining. A study carried out by Frirdich et al. (2019) observed that Campylobacter jejuni can change its shape from helical to coccoid when there was an unfavourable condition to survive. That's why coccoid shaped isolates are also screened in this present study. All 54 isolates confirmed as G-ve curved rods/s-shaped/coccoid in shape were initially checked for the oxidase test to screen oxidasepositive isolates and 23 isolates had shown oxidase-positive reaction (Fig. 3B). Out of which 8 raw milk isolates, 9 faecal swab isolates, 3 isolates each from dairy waste water and dairy milk filters had shown oxidasepositive reaction. In case of catalase test, only 15 isolates had shown catalase enzyme activity through the production of efferve scence in the presence of Hydrogen peroxide (Fig. 3C). These isolates were from different sources, such as 5 each from raw milk and faecal swab samples followed by 3 isolates from dairy wastewater and 2 isolates from dairy milk filter. None of the isolates from freshwater samples showed catalase test positive reaction. Total of 15 catalase positive isolates were further evaluated formicro-aerobic growth at $25^{\circ} \mathrm{C}$ wherein only
3 isolates showed no growth on Hi-Chrome $\mathrm{CAB}$ and suspected as Campylobacter isolates (Fig. 3D). Among three isolates, one isolate from raw milk and two from the faecal swab sample were suspected as Campylobacter species. While, none of the samples from freshwater, dairy wastewater, human handler swab, and dairy milk filter were confirmed by this test. Other 51 isolates among 54 isolates from raw milk and faecal swab samples such as freshwater, dairy wastewater, human handler swab, and milk filter swab which were confirmed based on Grams reaction, none of them showed characteristic results of catalase, oxidase and micro-aerobic growth at $25^{\circ} \mathrm{C}$. All 3 isolates were further evaluated for $\mathrm{H}_{2} \mathrm{~S}$ production and hippurate hydrolysis for tentative species identification. Wherein, 2 of the isolates showed hippurate hydrolysis with no $\mathrm{H}_{2} \mathrm{~S}$ production and was considered as C. jejuni. The remaining one isolate showed no hippurate hydrolysis along with $\mathrm{H}_{2} \mathrm{~S}$ production and it was tentatively indicated as $C$. coli. From the hippurate hydrolysis and $\mathrm{H}_{2} \mathrm{~S}$ production test (Fig. 3E and Fig. 3F), it was evident that among three isolates, 2 isolates might be $C$. jejuni and third isolate might be $C$. coli. The suspected isolates were mainly isolated from raw milk and faecal swab sample and they showed $\mathrm{H}_{2} \mathrm{~S}$ production test negative and hippurate hydrolysis test positive. In this study, out of 91 isolates selected initially, two were tentatively identified as $C$. jejuni (2.19\%) while one was identified as Campylobacter coli (1.0\%). With respect to the total no. of samples screened, $1.71 \%$ overall occurrence of Campylobacter species were observed including $1.14 \%$ of $C$. jejuni and $0.57 \%$ of $C$. coli. In case of individual samples types, the occurrence rate of $C$. jejuni in raw milk was found to be $3.33 \%(1 / 30)$ and $4 \%(1 / 25)$ for faecal swab samples. Similarly, a 4\% (1/25) occurrence rate of $C$. coli was observed in the faecal swab samples but there was a zero percentage (0/30) occurrence rate was observed in raw milk. While in freshwater, dairy wastewater, human handler swab, and dairy milk filter, zero percent occurrence was found for both $C$. jejuni and C. coli. 
Indian Journal of Animal Health, Special Issue, December, 2021

Campylobacter in dairy supply chain

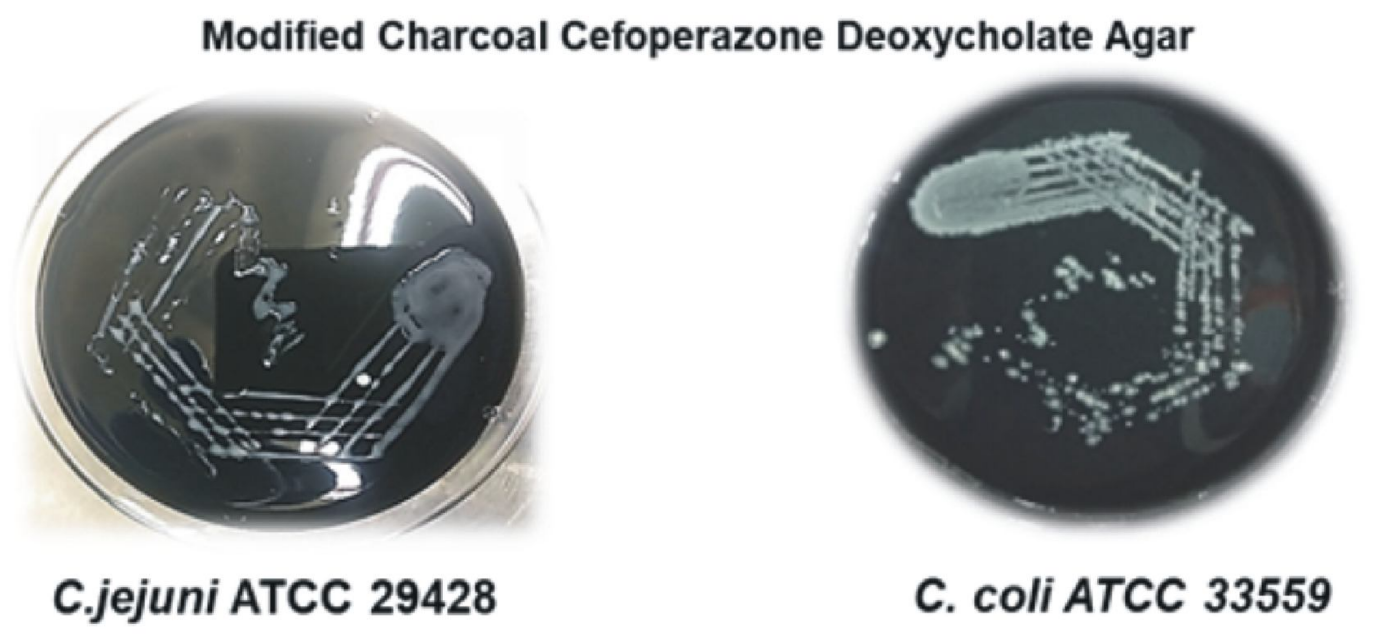

Blood containing Campylobacter agar (CAB)

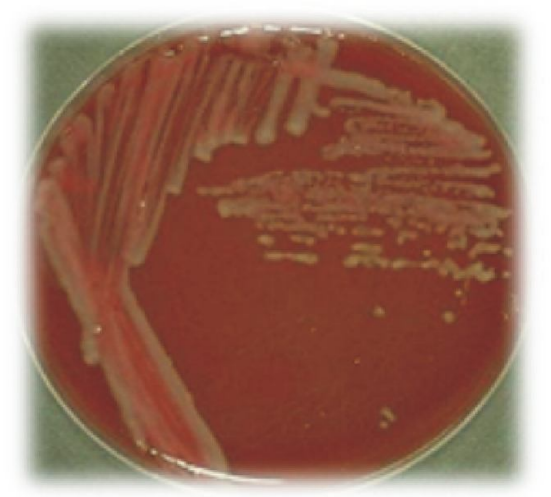

C.jejuni ATCC 29428

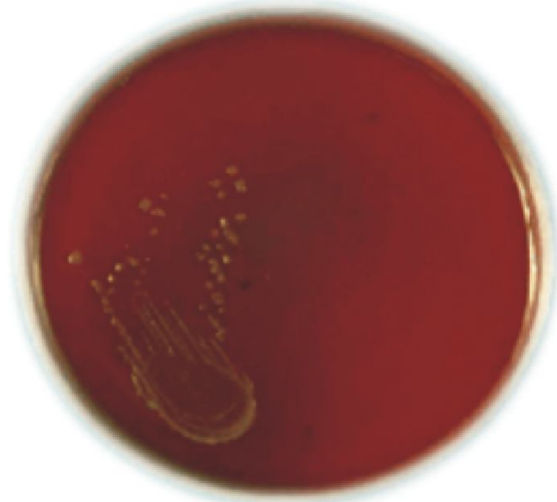

C. coli ATCC 33559

Fig. 1. The growth of Campylobacter species on mCCDA and CAB medium. The greyish, flat, characteristics colonies of Campylobacter on black colored mCCDA media and small, grayish in coloration, flat with irregular edges, non-hemolytic, mucoid colonies on blood containing $\mathrm{CAB}$ 
Indian Journal of Animal Health, Special Issue, December, 2021

Campylobacter in dairy supply chain

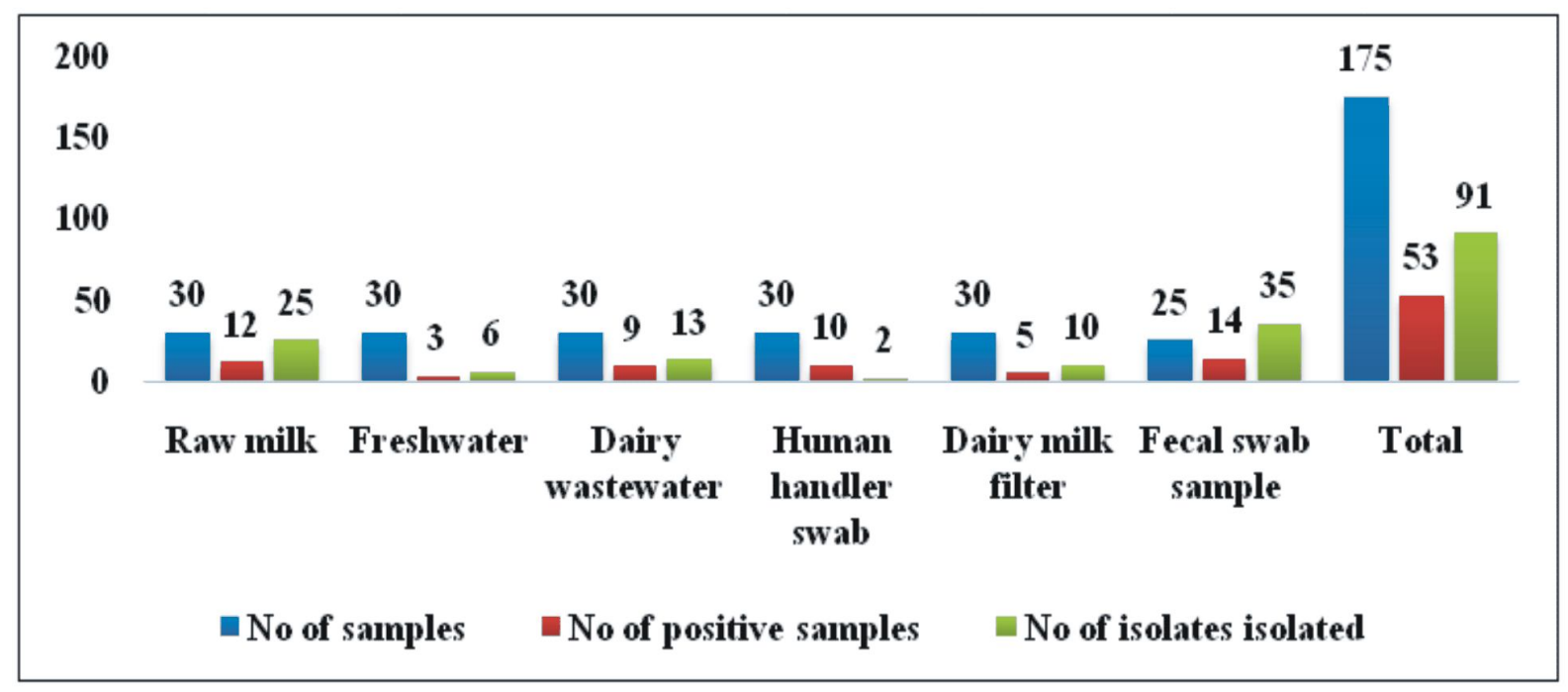

Fig. 2. Suspected isolates/colonies selected for biochemical confirmation

A
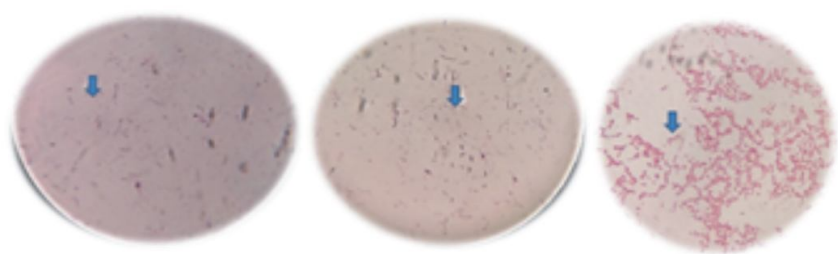

B

C
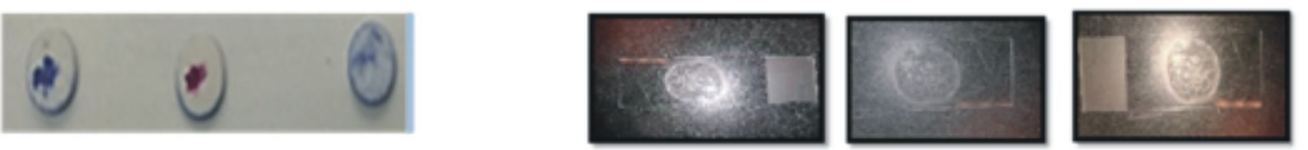

E
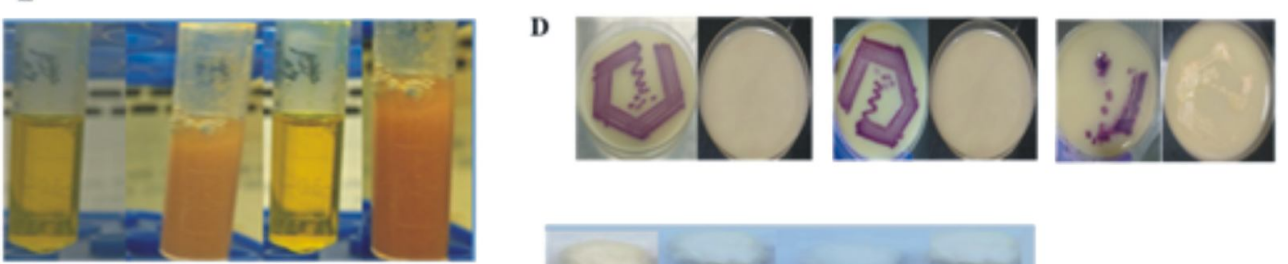

F

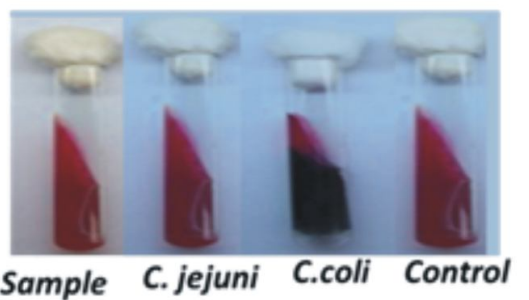

Fig. 3. Identification of Campylobacter species by phenotypic methods A. Grams staining - Gramnegative curved rods/s-shaped/coccoid in shape, B. Oxidase test, C. Catalase test, D. Microaerobic growth at $25^{\circ} \mathrm{C}$, E. Hippurate hydrolysis test, $\mathrm{F} . \mathrm{H}_{2} \mathrm{~S}$ production test 


\section{DISCUSSION}

To our acquaintance, this research work has given the very information regarding the occurrence of Campylobacter species in dairy supply chain. Initially, selective medium was selected based the characteristic growth of Campylobacter on commonly available selective medium based on their characteristic growth and convince of use in the laboratory. Therefore, the mCCDA media was selected for the further isolation of Campylobacter from the dairy supply chain. In a study by Jones (2003), two selective media were compared for isolation of Campylobacter species from stool samples which included blood containing medium (CampyBAP) and the other was blood-free medium (CCDA) and they found CCDA would be a superior medium as compared to the blood containing CampyBAP. The superiority of mCCDA media may be due to supplementation of medium with polymixin B and it has yielded higher isolation rate and greater selectivity as compared to Campylobacter isolation agar (Chon et al., 2012). Isolation and identification of Campylobacter was done on the basis of cultural and biochemical methods. About 91 characteristic colonies were selected from the mCCDA medium followed biochemical confirmation isolates colonies based on Grams reaction, catalase oxidase test followed by hippurate hydrolysis test and $\mathrm{H}_{2} \mathrm{~S}$ production. Finally, three isolates have phenotypically confirmed as Campylobacter spp. which were catalase-positive, oxidase-positive and no growth at $25^{\circ} \mathrm{C}$ under micro-aerobic conditions. Later, all 3 isolates were further evaluated for the $\mathrm{H}_{2} \mathrm{~S}$ production and Hippurate hydrolysis test to differentiate between $C$. jejuni and $C$. coli. Two isolates showed positive hippurate hydrolysis test and negative $\mathrm{H}_{2} \mathrm{~S}$ production, while one isolate had shown negative hippurate hydrolysis test and positive $\mathrm{H}_{2} \mathrm{~S}$ production. Based on the hippurate hydrolysis test and $\mathrm{H}_{2} \mathrm{~S}$ production, it was confirmed that two isolates as $C$. jejuni and one $C$. coli. The overall, 1.71 percent presence of Campylobacter was observed in 175 samples from the dairy supply chain. The occurrence rate of Campylobacter species in raw milk and fecal swab samples was found to be $3.33 \%(1 / 30)$ and $4 \%(2 / 25)$, respectively. Hence the occurrence of Campylobacter was found to be more in the fecal swab as compared to the raw milk sample. Except for raw milk and fecal swab sample, none of the samples had shown the occurrence of Campylobacter. Out of three biochemically confirmed Campylobacter isolates, two confirmed as Campylobacter jejuni and one as Campylobacter coli by using $\mathrm{H}_{2} \mathrm{~S}$ and hippurate hydrolysis test. From the two biochemically confirmed isolates of fecal swab samples, one was tentatively identified as $C$. jejuni and other Campylobacter coli, while the one positive isolate from raw milk was tentatively identified as C. jejuni. While in freshwater, dairy wastewater, human handler swab, and dairy milk filter, zero percent occurrence was found for both $C$. jejuni and $C$. coli. These results are very well supported by Wysok et al. (2011) who reported the presence of Campylobacter in milk from the selected regions of Poland. Out of 150 bulk milk samples, 7 samples were found positive. The occurrence rate of Campylobacter in milk samples found $4.6 \%$ which was more than the occurrence rate in milk in the present study $(1.71 \%)$. A total of 62 raw bulk tank milk samples were processed and only a single sample was found positive. The occurrence rate of Campylobacter was found at $1.6 \%$ which was lower than the occurrence rate in our current study. The occurrence rate of the present study was lower than the reports of Modi et al. (2015), who reported $2.91 \%$ occurrence rate in raw milk samples and the positive isolates are found to be $C$. jejuni. Hussain et al. (2007) reported an occurrence rate of Campylobacter in raw milk samples collected from three big cities of Pakistan with $10.2 \%$ prevalence which was higher than the prevalence rate in the current study results. Elango et al. (2009) reported $1.44 \%$ prevalence in raw milk samples. From 2008 to 2011, Giacometti et al. (2012) presented a prevalence data by monitoring four selected foodborne pathogens such as Salmonella, L.monocytogenes, E.coli, and C. jejuni from official microbiological records, these were 
detected by Real-time PCR in raw milk sold by self machines in seven Italian regions $(60,907$ samples from 1,239 vending machines). Campylobacter jejuni was the second most prevalent pathogen with $0.09 \%$ prevalence rate in milk samples with a significant increase in C. jejuni prevalence was observed over the years of the study. In Nigeria, Salihu et al. (2010) reported $12.9 \%$ prevalence rate of Campylobacter spp. in dairy faecal swab samples of cattle.

Based on the above finding, it is concluded that the prevalence of Campylobacter was more observed in the faecal swab samples than raw milk samples based on phenotypic methods.

Conflict of interest: Authors have no conflict of interest in this study.

\section{REFERENCES}

Barr W and Smith A, 2014. Acute diarrheal in adults. Am Fam Physician, 89(3): 180-189

Bintsis T, 2017. Food-borne pathogens. AIMS Microbiol, 3(3): 529-563, doi: 10.3934/ microbiol.2017.3.529

Chon JW, Hyeon JY, Yim JH, Kim JH, Song KY et al., 2012. Improvement of modified charcoalcefoperazone-deoxycholate agar by supplementation with a high concentration of polymyxin B for detection of Campylobacter jejuni and $C$. coli in chicken carcass rinses. Appl Environ Microbiol, 78(5): 1624-1626, doi: 10.1128/ AEM.07180-11

Dhanashekar R, Akkinepalli S and Nellutla A, 2012. Milk-borne infections. An analysis of their potential effect on the milk industry. Germs, 2(3): 101-109, doi: 10.11599/germs.2012.1020

Elango A, Dhanalakshmi B, Pugazhenthi TR, Jayalalitha V, Kumar CN et al., 2009. PCRrestriction fragment length polymorphism of Campylobacter jejuni isolates. Egypt J Dairy Sci, 37(2): 69-173

Facciolà A, Riso R, Avventuroso E, Visalli G, Delia SA et al., 2017. Campylobacter: from microbiology to prevention. J Prev Med Hyg, 58(2): E79-E92

Frirdich E, Biboy J, Pryjma M, Lee J, Huynh S et al., 2019. The Campylobacter jejuni helical to coccoid transition involves changes to
Author's contribution: SB, DB, and HVR: Conceived and design the study, carried out the sampling and worked on the isolation and phenotypic identification of Campylobacter in dairy supply chain; AJ, HVR, NK, and HMR: Performed the data analysis and prepareded the manuscript.

\section{ACKNOWLEDGEMENTS}

This work was carried out under the Early Carrier Research Scheme funded by Science and Engineering Research Board (SERB), Department of Science and Technology (DST), Ministry of Science and Technology, Govt. of India. The Authors gratefully acknowledge the Director, ICAR-NDRI for providing the necessary infrastructure facility to carry out this research.

peptidoglycan and the ability to elicit an immune response. Mol Microbiol, 112(1): 280-301, doi: 10.1111/mmi.14269

Giacometti F, Serraino A, Finazzi G, Daminelli P, Losio MN et al., 2012. Sale of raw milk in northern Italy: food safety implications and comparison of different analytical methodologies for detection of foodborne pathogens. Foodborne Pathog Dis, 9(4): 293-297, doi:10.1089/fpd.2011.1052

Hussain I, Mahmood MS, Akhtar M and Khan A, 2007. Prevalence of Campylobacter species in meat, milk and other food commodities in Pakistan. Food Microbiol, 24(3): 219-222, doi: 10.1016/ j.fm.2006.06.001

Igwaran A and Okoh AI, 2019. Human campylobacteriosis: A public health concern of global importance. Heliyon, 5(11): e02814, doi: 10.1016/j.heliyon.2019.e02814

ISO 10272-1, 2006. Microbiology of food and animal feeding stuffs - Horizontal method for detection and enumeration of Campylobacter spp. - Part 1: Detection method

Kaakoush NO, Castaño-Rodríguez N, Mitchell HM and Man SM, 2015. Global epidemiology of Campylobacter infection. Clin Microbiol Rev, 28(3): 687-720, doi:10.1128/CMR.00006-15

MARD (Minister of Agriculture and Rural Development), 2007. Regulation of the Minister of Agriculture and Rural Development from 29 
December 2006- On veterinary requirements at the production of products of the animal origin intended direct sales. Journal of Laws, No 5, item 38, Government Legislation Centre, Warsaw, Poland

Mitruka BM, 2017. Methods of Detection and Identification of Bacteria (1977). CRC Press, Taylor \& Francis Group, USA

Modi S, Brahmbhatt MN, Chatur YA and Nayak JB, 2015. Prevalence of Campylobacter species in milk and milk products, their virulence gene profile and anti-bio gram. Vet World, 8(1): 1-8, doi: 10.14202/vetworld.2015.1-8

Salihu MD, Junaidu AU, Magaji AA and Rabiu ZM, 2010. Study of Campylobacter in raw cow milk in Sokoto State, Nigeria. Br J Dairy Sci, 1(1): 1-5

Same RG and Tamma PD, 2018. Campylobacter infections in children. Pediatr Rev, 39(11): 533-
541, doi: 10.1542/pir.2017-0285

Sippy R, Sandoval-Green CM, Sahin O, Plummer P, Fairbanks WS et al., 2012. Occurrence and molecular analysis of Campylobacter in wildlife on livestock farms. Vet Microbiol, 157(3-4): 369375, doi: 10.1016/j.vetmic.2011.12.026

Smith S, Meade J, McGill K, Gibbons J, Bolton D et al., 2015. Restoring the selectivity of modified charcoal cefoperazone deoxycholate agar for the isolation of Campylobacter species using tazobactam, a $\beta$-lactamase inhibitor. Int $\mathbf{J}$ Food Microbiol, 210: 131-135, doi: 10.1016/ j.ijfoodmicro.2015.06.014

Wysok B, Wiszniewska-£aszczych A, Uradziñski J and Szteyn J, 2011. Prevalence and antimicrobial resistance of Campylobacter in raw milk in the selected areas of Poland. Pol J Vet Sci, 14(3): 473477, doi:10.2478/v10181-011-0070-3

Received-23.08.2021, Accepted-12.11.2021, Published-01.12.2021

Section Editor: Prof. M. K. Sanyal, Member, Editorial Board 